ARTICLE

Received 18 Jul 2016 | Accepted 10 Nov 2016 | Published 20 Dec 2016

DOI: $10.1038 /$ ncomms13904 OPEN

\title{
Conformation-driven quantum interference effects mediated by through-space conjugation in self-assembled monolayers
}

Marco Carlotti ${ }^{1}$, Andrii Kovalchuk ${ }^{1}$, Tobias Wächter ${ }^{2}$, Xinkai Qiu$^{1}$, Michael Zharnikov ${ }^{2}$ \& Ryan C. Chiechi ${ }^{1}$

Tunnelling currents through tunnelling junctions comprising molecules with cross-conjugation are markedly lower than for their linearly conjugated analogues. This effect has been shown experimentally and theoretically to arise from destructive quantum interference, which is understood to be an intrinsic, electronic property of molecules. Here we show experimental evidence of conformation-driven interference effects by examining through-space conjugation in which $\pi$-conjugated fragments are arranged face-on or edge-on in sufficiently close proximity to interact through space. Observing these effects in the latter requires trapping molecules in a non-equilibrium conformation closely resembling the $\mathrm{X}$-ray crystal structure, which we accomplish using self-assembled monolayers to construct bottom-up, large-area tunnelling junctions. In contrast, interference effects are completely absent in zero-bias simulations on the equilibrium, gas-phase conformation, establishing through-space conjugation as both of fundamental interest and as a potential tool for tuning tunnelling charge-transport in large-area, solid-state molecular-electronic devices.

\footnotetext{
${ }^{1}$ Stratingh Institute for Chemistry \& Zernike Institute for Advanced Materials, University of Groningen, Nijenborgh 4, Groningen 9747 AG, The Netherlands. ${ }^{2}$ Applied Physical Chemistry, Heidelberg University, Im Neuenheimer Feld 253, Heidelberg 69120, Germany. Correspondence and requests for materials should be addressed to R.C.C. (email: r.c.chiechi@rug.nl).
} 
central challenge in molecular electronics is uncertainty in the conformation and binding geometry of a molecule between two electrodes. Small differences exert large effects on tunnelling charge-transport ${ }^{1}$, yet it is this sensitivity that drives scientific interest because functionality arises from precise control over conformation and geometry ${ }^{2-4}$. Nature accomplishes this level of control through self-assembly, in which molecular systems can be arranged precisely enough to support long-range electron transfer over several microns ${ }^{5}$. In selfassembled monolayers (SAMs), molecules are fixed in a specific conformation and binding geometry, arranging themselves in ordered, two-dimensional crystal-like domains. Tunnelling junctions comprising SAMs, therefore, fix molecules in a specific conformation and binding geometry that defines the smallest dimension of the junction, through which charges tunnel; they are a form of bottom-up nanotechnology 6,7 . The effects of conformational confinement on molecular charge-transport are particularly interesting in the case of $\pi$-conjugated molecules because conductivity (hopping) and transmission (tunnelling) are strongly related to the extent of electronic delocalization. Quantum interference (QI) effects ${ }^{8}$ arising from cross-conjugation in such conjugated molecules have been demonstrated experimentally ${ }^{9,10}$, but they have also been predicted for $\pi$ systems that are close enough in space to interact $^{11}$. Unlike for conjugation patterns, which are an intrinsic property, this type of QI is extremely sensitive to conformation; the two $\pi$ systems must be precisely aligned to form a through-space conjugation interaction.

Single-molecule conductance techniques are not well suited for observing effects that require precise control over conformation because they apply force to molecules and the geometry of the junction differs with each observation ${ }^{12,13}$. And although SAMs are crystalline and can be highly ordered over small areas, defects and grain boundaries can influence the current-density versus voltage $(J / V)$ properties in large-area $\left(\mu \mathrm{m}^{2}\right)$ measurements ${ }^{14}$. In addition to these experimental challenges, distinguishing between destructive QI and differences in conductance arising from conjugation length require fortuitous level-alignment in the assembled junction ${ }^{15}$. Through-space QI is potentially useful beyond validating theoretical predictions as it could couple small structural or conformational changes (for example, from external mechanical forces) into exponential changes in conductance. It is also synthetically advantageous because cross-conjugation tends to involve heteroatoms, which can introduce unexpected and complex electrostatic effects ${ }^{16}$.

Here we describe through-space destructive QI effects in tunnelling charge-transport through SAMs comprising molecules in which the central aromatic rings are spatially separated by saturated methylene bridges. These effects are consistently present in simulated transmission spectra derived from the X-ray crystal structures of the compounds used to form the SAMs, but not in minimized, gas-phase geometries. We resolved these effects experimentally by conducting-probe atomic force microscopy (CP-AFM) and in large-area junctions using eutectic Ga-In (EGaIn) as a top-contact ${ }^{17}$. These results prove that the $J / V$ characteristics are not the result of defects and demonstrate both that a single conformation resembling that of the bulk crystal dominates transport in a SAM, mitigating the problem of uncertainty in the geometry of tunnelling junction, and that symmetric Au contacts can be used to model electronic effects qualitatively in large-area junctions with EGaIn top-contacts.

\section{Results}

Conformation within the SAM. We investigated tunnelling transport in systems characterized by two phenyl rings in either a vertical, face-on ( $p$ seudo-p-bis((4-(acetylthio)phenyl)ethynyl)- $p$ - [2,2]cyclophane, PCP) or edge-on (2,6-bis(((4-acetylthio)phenyl) ethynyl)-9,10-dihydroantracene, $\mathrm{AH}$ ) arrangement and that are held in close proximity with saturated ethylene bridges in $\mathrm{Au}^{\mathrm{TS}} / \mathrm{SAM} / / \mathrm{EGaIn}$ and $\mathrm{Au}^{\mathrm{M}} / \mathrm{SAM} / / \mathrm{Au}^{\mathrm{AFM}}$ junctions (where '/" and ' $/ /$ ' denote covalent and van der Waals contacts, respectively, $\mathrm{Au}^{\mathrm{TS}}$ is template-stripped $\mathrm{Au}, \mathrm{Au}^{\mathrm{M}}$ is $\mathrm{Au}$-on-mica and $\mathrm{Au} \mathrm{AFM}^{\mathrm{A}}$ is a Au-coated AFM tip.) In both cases the precise alignment of the $\pi$ systems is controlled by packing in the SAM on the Au substrates. We measured 1,4-bis(((4-acetylthio)phenyl)ethynyl) benzene (OPE3) and 2,6-bis(((4-acetylthio)phenyl)ethynyl) antracene $(\mathrm{AC})^{18}$ to compare molecules with the same end-toend lengths as $\mathrm{PCP}$ and $\mathrm{AH}$, but that are conjugated throughbond; the structures and tunnelling pathways are shown in Fig. 1. Edge-on transport is difficult to address experimentally because at room temperature $\mathrm{AH}$ is in rapid equilibrium between bent and planar conformers in solution and in the gas phase, preferring the bent conformation by $3.5 \mathrm{kcal} \mathrm{mol}^{-1}$ (see Supplementary Table 1 and Supplementary Fig. 1). Thus, the bent conformer would dominate when $\mathrm{AH}$ is trapped in a single-molecule junction. In the crystalline state, however, $\mathrm{AH}$ adopts a planar conformation to minimize the free volume. To establish experimentally the precise spacial arrangements of the phenyl rings comprising the through-space elements, we obtained the X-ray crystal structures of AH and PCP, which we denote as AH-crystal and PCP-crystal. These structures and data are shown in Supplementary Figs 2 and 3 and Supplementary Table 2.

We determined the tunnelling transport properties by comparing the magnitude of $\log |J|$ in $\mathrm{Au}^{\mathrm{TS}} / \mathrm{SAM} / / \mathrm{EGaIn}$ junctions comprising SAMs of OPE3, PCP, AH and AC, which we grew from symmetric bis-thioacetates via in situ deprotection (see 'Methods' section) ${ }^{9,19}$. We characterized the SAMs by ellipsometry, high-resolution X-ray photoelectron spectroscopy (HRXPS) and near-edge X-ray absorption fine structure (NEXAFS) spectroscopy. These data are summarized in Table 1 along with literature and benchmark values for SAMs of $\mathrm{CH}_{3}\left(\mathrm{CH}_{2}\right)_{17} \mathrm{~S}$ (SC18) for comparison. The spectra are shown in Supplementary Figs 4 and 5. The principal difference appears to be the tilt angles, which are slightly higher for $\mathrm{Au}^{\mathrm{TS}}$ than $\mathrm{Au}^{\mathrm{TM}}$, thus the SAMs are slightly thinner (except for AH), but still quite dense (on the order of $10^{14}$ molecules per $\mathrm{cm}^{2}$ ). The S $2 \mathrm{p}$ X-ray photoelectron spectra of the SAMs exhibited characteristic signals of thiolate and unbound SH/SAc groups, with much higher intensity of the latter signals, because of the differences in the attenuation $^{20}$. These spectra suggest that the molecules are indeed assembled upright in the SAMs, with one of the terminal sulfur atoms bound to the substrate and another one exposed to the SAM-ambient interface, where it can be contacted by EGaIn or Au. Upright molecular orientation is also apparent from the NEXAFS spectra, which exhibited quite small but distinct linear dichroism corresponding to an average molecular tilt angle of $\sim 40^{\circ}$. In addition, the spectra exhibited the characteristic absorption resonances of OPE-like compounds (Supplementary Note 1), establishing the identity of the AC, AH, OPE3 and PCP films. Significantly, there were no traces of contamination, including a lack of the very pronounced resonance of carboxyl moieties (most frequent contamination), demonstrating the high purity of the prepared monolayers. Measuring well characterized, high-quality SAMs is paramount as, unlike top-down, singlemolecule techniques (break junctions and so on) or few molecules techniques (conducting-probe AFM and so on.), EGaIn is a bottom-up, large-area technique ${ }^{7}$ and is therefore sensitive to the detailed structure of the SAM because it defines the physical shape of the junction and the EGaIn//SAM interface ${ }^{21-23}$.

Charge-transport characteristics. In through-space conjugation two $\pi$ systems are held close enough in space to interact without 
a

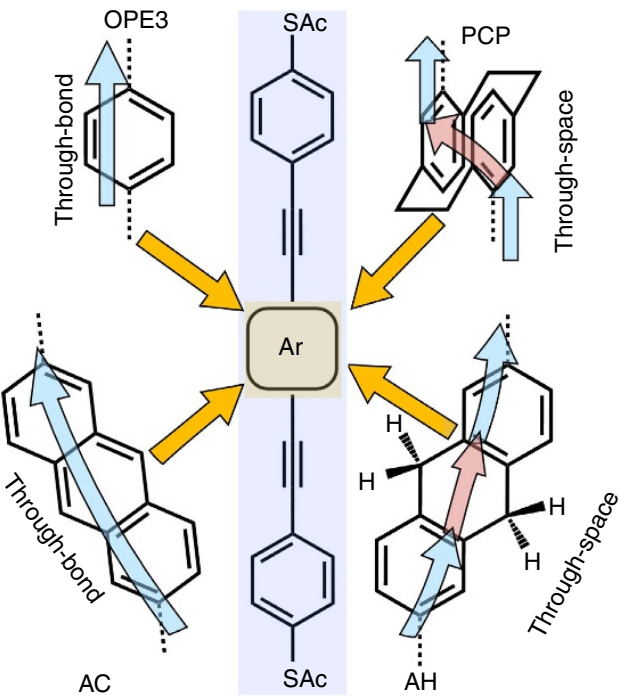

b
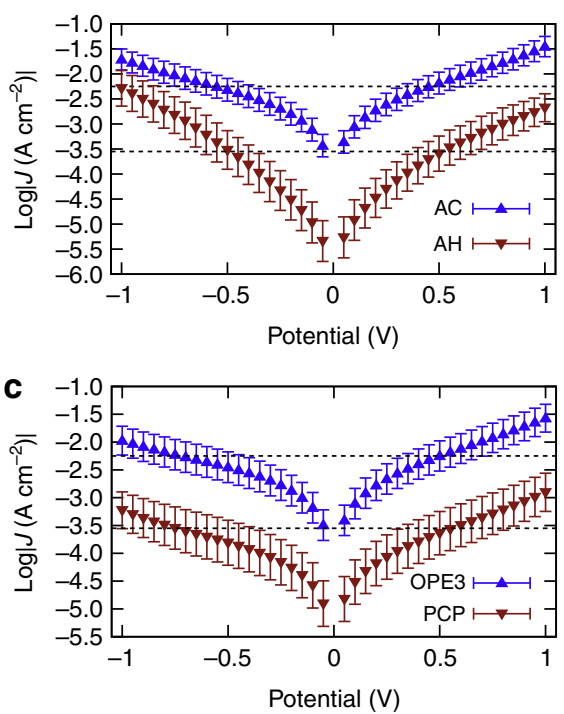

Figure 1 | Molecular conformation effects on charge transport in tunnelling junctions. (a) The structures of the compounds from which SAMs were grown on $\mathrm{Au}^{\mathrm{TS}}$. The blue arrows show through-bond pathways and the red arrows through-space pathways. (b) Current-density versus voltage plots of $\mathrm{Au}^{\mathrm{TS}} / \mathrm{SAM} / / \mathrm{EG}$ aln junctions of AH (red triangles) and AC (blue triangles) and (c); PCP (red triangles) and OPE3 (blue triangles). The dashed lines are to guide the eyes. Each data point is the peak of a Gaussian fit of log-normal plots of $|J|$ for that voltage. Error bars are confidence intervals. Tunnelling junctions were formed by contacting grounded $\mathrm{Au}^{\mathrm{TS}} / \mathrm{SAM}$ substrates with sharp tips of EGaln and applying a potential.

Table 1 | Summary of SAMs characterization and $\mathrm{Au}^{\mathrm{TS}} / \mathrm{SAM} / /$ EGaln junctions properties.

\begin{tabular}{|c|c|c|c|c|c|}
\hline Compound & AH & AC & PCP & OPE3 & SC18 \\
\hline XPS thickness $(\AA)$ & $23.9\left(19.0^{\star}\right)$ & $16.2\left(25.1^{\star}\right)$ & 15.8 & $13.7\left(17.5^{\dagger}\right)$ & 20.9 \\
\hline Ellipsometric thickness $\ddagger(\AA)$ & $25.4 \pm 0.5$ & $17.0 \pm 0.5$ & $14.6 \pm 0.4$ & $14.3 \pm 0.6\left(20.6^{\dagger}\right)$ & $20.2 \pm 0.5$ \\
\hline Density $\left(10^{14}\right.$ molecules per $\mathrm{cm}^{2}$ ) & 4.7 & 4.6 & 3.9 & 3.5 & 4.6 \\
\hline $\log |J| @ 0.5 \vee\left(\mathrm{Acm}^{-2}\right)$ & $-3.37 \pm 0.84$ & $-2.18 \pm 0.44$ & $-3.63 \pm 0.80$ & $-2.27 \pm 0.30$ & $-4.96 \pm 0.87$ \\
\hline Yield of working junctions (\%) & 96 & 98 & 94 & 92 & 79 \\
\hline Number of working junctions & 55 & 56 & 75 & 60 & 28 \\
\hline Number of traces & 550 & 560 & 750 & 600 & 280 \\
\hline
\end{tabular}

the aid of an underlying $\sigma$ framework. Electronic overlap and, therefore, tunnelling charge-transport is extraordinarily sensitive to the conformation imposed by the junction geometry because there is no rigid framework to keep $\pi$ systems aligned and their relative displacement is subject to interactions with neighbouring molecules. This sensitivity is why through-space QI effects are difficult to resolve experimentally; for example, destructive QI is predicted in stacked benzene rings, but only through specific pathways and precise arrangements of the two rings ${ }^{11}$. Previous break-junction measurements on $p$-2,2-cyclophane moieties (which are similar to $\mathrm{PCP}$ ) probed tunnelling charge-transport perpendicular to the plane of the phenyl rings (that is, down the stack $)^{24}$. And the conductance of $\mathrm{AH}$ was higher than AC in break-junctions, where the former exhibited a decrease in conductance with increasing electrode separation ${ }^{10}$. No QI was predicted or observed in either of these studies; that is, an electron tunnelling through space (the red arrows in Fig. 1a) is not itself a predictor of QI or of different magnitudes of tunnelling transport compared with their through-bond analogues.

Figure $1 \mathrm{~b}, \mathrm{c}$ show the $J / V$ curves for $\mathrm{AC}, \mathrm{AH}, \mathrm{OPE} 3$ and PCP; the histograms from which these data were derived are shown in Supplementary Fig. 6. The SAMs on $\mathrm{Au}^{\mathrm{TS}}$ are robust enough to scan to $\pm 1 \mathrm{~V}$, revealing the onset of asymmetric conductivity $(\log |J| @ 1 \mathrm{~V} \neq \log |J| @-1 \mathrm{~V})$ in $\mathrm{AH}$, possibly because the two halves of $\mathrm{AH}$ approach resonance independently of each other due to the disparate contacts ${ }^{25}$. Data from $\mathrm{Au}^{\mathrm{M}} / \mathrm{SAM} / \mathrm{Au}^{\mathrm{AFM}}$ junctions acquired using CP-AFM show the same trends in conductance, although we could not resolve the low-bias regions of PCP or AH (presumably because they are below the detection limit; Supplementary Figs 7 and 8.) Nonetheless, these data confirm that the trend is not due to defects in the SAM (the contact area of CP-AFM is on the order of dozens of molecules) or the influence of the $\mathrm{Ga}_{2} \mathrm{O}_{3}$ layer on the EGaIn electrode. Both the $\mathrm{Au}^{\mathrm{M}} / \mathrm{SAM} / \mathrm{Au}^{\mathrm{AFM}}$ and $\mathrm{Au}{ }^{\mathrm{TS}} / \mathrm{SAM} / \mathrm{EGaIn}$ junctions of OPE3 and $\mathrm{AC}$ exhibit larger magnitudes of $J$ by about a factor of 100 than PCP and $\mathrm{AH}$, respectively (which may be a sign that destructive QI is dominant in the latter two SAMs); however, unambiguously ascribing transport data to destructive QI effects is difficult because the observable is smaller magnitudes of $\log |J|$, which is sensitive to myriad of factors ${ }^{26}$. Thus, we applied various tests for QI (see Supplementary Notes 2 and 3 and Supplementary Figs 9-16); it is predicted for PCP and AH, but not OPE3 or AC, in agreement with the $J / V$ data in Fig. 1. One of these tests is the product of the coefficients of the frontier orbitals on the termini 
of a molecule; if they are the same, destructive QI is predicted between those orbitals ${ }^{8}$. This test is shown in Fig. 2 for $\mathrm{AH}$ and AH-crystal. The change in the signs of the orbital coefficients between $\mathrm{AH}$ and $\mathrm{AH}$-crystal is accompanied by an increase in distance between the phenyl rings of $0.06 \AA$, at which point the two halves (separated by the $\mathrm{CH}_{2}$ bridges) become almost coplanar, aligning the two phenyl $\pi$-systems and allowing for better electronic overlap. In this conformation the two phenyl rings are connected by through-space conjugation and this form of through-space conjugation apparently induces QI analogously to the face-on arrangement in PCP; however, QI has not previously been predicted for $\mathrm{AH}$ because only the gas-phase minimized structure has been considered. Destructive QI may be 'switched on' in by small changes in conformation.

\section{Discussion}

For further insight into the predicted transport properties, we simulated transmission spectra for isolated molecules of $\mathrm{AC}, \mathrm{AH}$, OPE3 and PCP bound to Au electrodes. To isolate relative effects of molecular structure, we performed density function theory (DFT) calculations with 12-atom $\mathrm{Au}(111)$ clusters bound to the terminal sulfurs at hexagonal close-pack hollow sites. These calculations are not models of $\mathrm{Au}^{\mathrm{TS}} / \mathrm{SAM} / / \mathrm{EGaIn}$ or $\mathrm{Au}^{\mathrm{M}} / \mathrm{SAM} /$ $\mathrm{Au}{ }^{\mathrm{AFM}}$ junctions, rather they are computational experiments on idealized systems to isolate the effects of electronic structure and conformation on zero-bias transmission by using single molecules bound to small clusters of Au. There are numerous collective effects in SAMs that can affect the electrostatics and level-alignment that cannot be captured by single-molecule calculations ${ }^{27-30}$. Without detailed knowledge of the packing of the molecules in the SAM and atomistic detail of the $\mathrm{Ga}_{2} \mathrm{O}_{3}$ electrode and $\mathrm{SAM} / / \mathrm{Ga}_{2} \mathrm{O}_{3} \mathrm{EGaIn}$ interface, none of which are

a

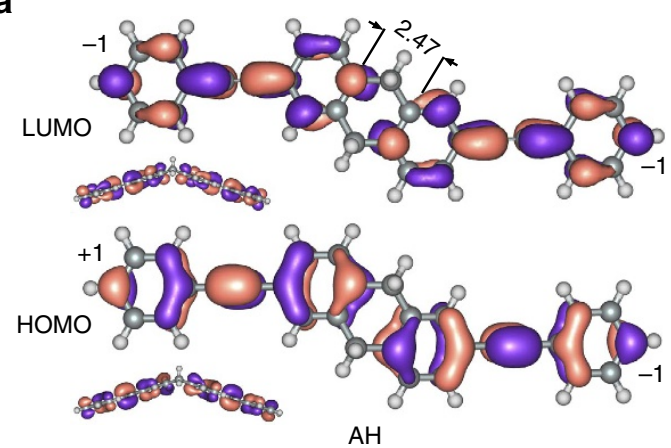

b

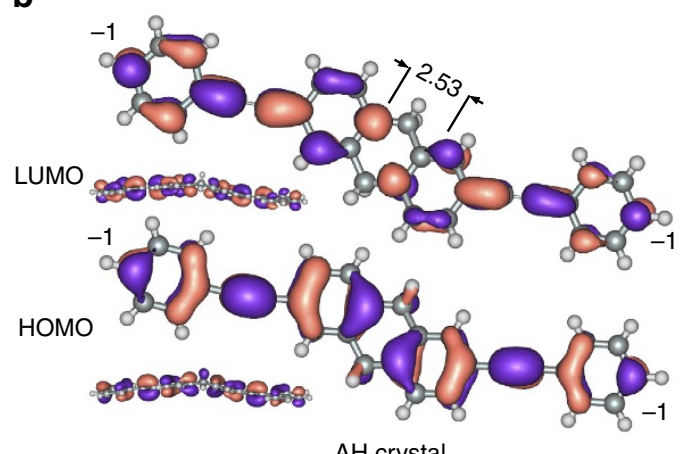

AH crystal

Figure 2 | Impact of molecular geometry on molecular orbitals. The $\mathrm{HOMO}$ and LUMO of $\mathrm{AH}$ (a) and $\mathrm{AH}$-crystal (b) with through-space distances indicated in $\AA$. The signs of the orbital coefficients at the terminal sites are shown at these sites. available, it is impossible to construct an accurate model capable of predicting these effects. The principal limitations of using a model $\mathrm{Au} / \mathrm{molecule} / \mathrm{Au}$ junction is that we cannot reliably estimate the Fermi level $E_{\mathrm{f}}$ and the relative level-alignments will not reflect any collective effects such as broadening or electrostatic shifts ${ }^{31}$ (for example, the $0.4-0.5 \mathrm{eV}$ shift induced by $\mathrm{S}-\mathrm{Au}$ bonds in $\mathrm{SAMs}^{32}$ ). However, we can still compare (similar) molecular structures and draw meaningful (qualitative) conclusions in combination with experimental data from $\mathrm{Au}^{\mathrm{TS}} / \mathrm{SAM} / / \mathrm{EGaIn}$ junctions ${ }^{9}$. We estimated $E_{\mathrm{f}}$ by adding the calculated highest-occupied $\pi$ state (HOPS) to the experimentally derived transition voltages ${ }^{31,33} V_{\text {trans }}$ for each $\mathrm{Au}^{\mathrm{TS}} / \mathrm{SAM} / / \mathrm{EGaIn}$ junction (see Supplementary Table 3 ) to establish the relative level-alignment between related structures (that is, OPE3 and PCP; $\mathrm{AH}$ and AC).

Figure $3 \mathrm{a}$ shows the resulting transmission spectra from DFT calculations for OPE3 and PCP Au/molecule/Au junctions. The U-shape centred at $\sim 0.5 \mathrm{eV}$ is indicative of transmission probabilities in the frontier orbital gap of the molecule. The lower overall transmission of and PCP and PCP-crystal is the result of the the break in conjugation at the cyclophane ring, that is, there is no formal resonance structure connecting the two halves of the molecule. The plots for PCP and PCP-crystal also exhibit sharp dips around $-1 \mathrm{eV}$, which is a sign of QI; at this energy the frontier orbitals interfere destructively and a node appears in the wave function, suppressing transmission sharply. The most pronounced differences between PCP and PCP-crystal are that the transmission is higher everywhere for the former and the energy and magnitude of the dip ascribed to QI shifts by $\sim 0.5 \mathrm{eV}$ (that is, the positions and lengths of the solid red arrows in Fig. 3a differ.) This observation highlights the utility and limitations of using zero-bias, single-molecule junctions as models for $\mathrm{Au}^{\mathrm{TS}} / \mathrm{SAM} / / \mathrm{EGaIn}$ junctions; both the overall conductance (the integrated transmission near $E_{\mathrm{f}}$ ) and the position of the QI feature are sensitive to minor conformational changes. Supplementary Fig. 17 compares some of these
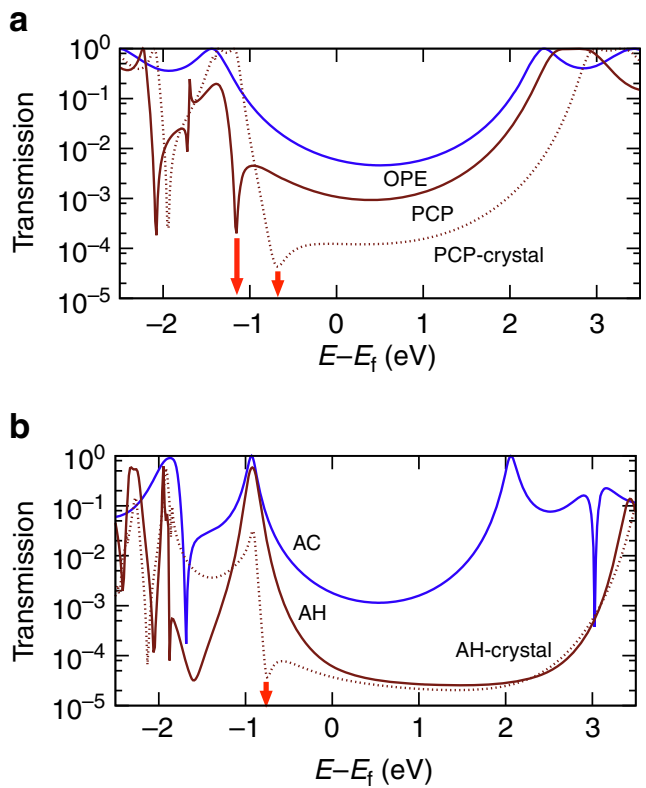

Figure 3 | Charge transmission probability plots. Transmission plots comparing, (a) PCP (red), OPE3 (blue) and PCP-crystal (dashed red) and (b) $\mathrm{AH}$ (red), $\mathrm{AC}$ (blue) and $\mathrm{AH}$-crystal. The 'crystal' suffix denotes X-ray crystal structures; the others are gas-phase minimized structures. The energy of the Fermi level $E_{f}$ was computed by adding $V_{\text {trans }}$ to the HOPS. Destructive QI features are marked with thick red arrows. 
differences, which are as small as $0.05 \AA$ in the cyclophane core. The net effect is that the $x$-axes in Fig. 3 as well as the relative position of the dip in transmission will be shifted in the actual junctions.

Figure $3 \mathrm{~b}$ shows the transmission spectra of $\mathrm{AC}$ and $\mathrm{AH}$ $\mathrm{Au} / \mathrm{molecule} / \mathrm{Au}$ junctions. Analagously to OPE3, AC shows a U-shaped curve centred at $\sim 0.5 \mathrm{eV}$; unlike OPE3 and PCP, however, the positive resonances (not QI) near the frontier orbitals for $\mathrm{AC}$ are closer to $0 \mathrm{eV}$ than they are for $\mathrm{AH}$ because there is a more pronounced change in the frontier orbital gap between linear and through-space conjugation in the edge-on case $(\mathrm{AH})$ than the face-on case (PCP.) It is tempting to ascribe this difference between the investigated systems to the lower conductivity of $\mathrm{AH}$ compared with $\mathrm{AC}$-one is conjugated and one is not--whereas cyclophanes exhibit similar properties to phenyl rings (for example, functional groups direct identical pseudo para/meta substitution). Fig. 1b,c, however, show that SAMs of PCP are experimentally much less conductive than SAMs of OPE3 across the entire bias window, while $\mathrm{AH}$ approaches the conductivity of AC, nearly crossing at $-1 \mathrm{~V}$. This behaviour is consistent with hypothesis that the conductance of $\mathrm{AH}$ in the low-bias regime is dominated by a sharp, destructive QI feature and that, as the bias is increased, transmission increases rapidly; that is, the dip in the dashed line (AH-crystal) in Fig. $3 \mathrm{~b}$ is shifted close to $E_{\mathrm{f}}$ in actual $\mathrm{Au}^{\mathrm{TS}} / \mathrm{SAM} / / \mathrm{EGaIn}$ junctions. The solid line in Fig. $3 \mathrm{~b}$ is the minimized, gas-phase conformation of $\mathrm{AH}$; unlike the case of PCP and PCP-crystal, the interference feature present for $\mathrm{AH}$-crystal vanishes entirely with $\mathrm{AH}$. We ascribe this difference to the changes in the frontier orbitals shown in Fig. 2 when AH adopts a planar conformation in the solid-state. This comparison does not imply that $\mathrm{AH}$-crystal reflects the exact conformation of $\mathrm{AH}$ in a SAM; however, if $\mathrm{AH}$ adopts a planar conformation in a bulk crystal, it is very likely that it does so when confined to a $2 \mathrm{D}$ molecular film on a solid substrate.

The extreme sensitivity of through-space QI effects on molecular conformation demonstrated by the DFT calculations
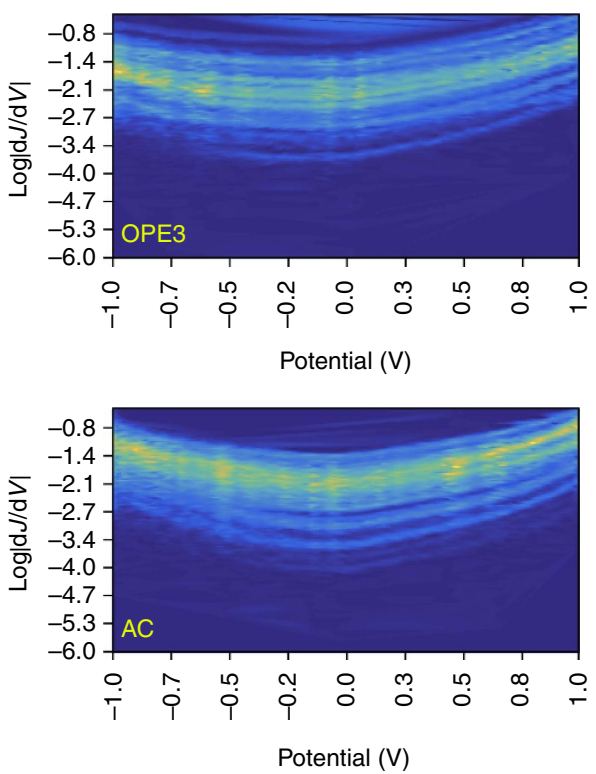

presents a significant experimental challenge that has confounded past efforts to characterize the effects of through-space conjugation on tunnelling charge-transport. Wire-like $p$-2,2-cyclophane moieties similar to PCP probed by crossed-wires, scanning tunnelling microscopy and CP-AFM in mixed-monolayers and sparse SAMs - that is, not in dense SAMs like those formed from PCP-exhibited only slightly suppressed conductance ${ }^{34,35}$. And attempts to compare edge-on through-space and through-bond conduction pathways (analogous to $\mathrm{AH}$ ) were similarly frustrated; although through-space interactions were found in the crystal structure, there was no correlation with conductance measurements in break-junctions ${ }^{36}$. The inconsistency of these observations may be a reflection of the sensitivity of through-space effects to conformation (or simply better experimental techniques). These results underscore the importance of considering the different geometries molecules adopt in solid, gas or dissolved phases and in single-molecule junctions and the challenges of growing densely packed SAMs from $\pi$-conjugated molecules.

We are confident in ascribing the low conductivity of SAMs of PCP to QI because it has been predicted for face-on throughspace transport ${ }^{11}$, however, the most robust experimental proof of destructive QI is the appearance of negative curvature in conductance heatmap plots of differential conductance $\log \left|\frac{d J}{d V}\right|$ versus $V$ (ref. 37). This curvature only appears if the energy of the interference in the assembled junction is close enough to $E_{\mathrm{f}}$ that the feature lies almost entirely within the bias window ${ }^{15}$. The dip in transmission for PCP and AH-crystal in Fig. 3 is far from $E_{\mathrm{f}}$, but (as described above) these calculations do not reflect the actual level-alignment in the $\mathrm{Au}^{\mathrm{TS}} / \mathrm{SAM} / / \mathrm{EGaIn}$ junctions. Differential conductance heatmaps of OPE3, AC, PCP and AH are shown in Fig. 4. These plots are constructed from histograms of $\log \left|\frac{\mathrm{d} J}{\mathrm{~d} V}\right|$ obtained from numerical derivatives of individual $J / V$ plots. (One advantage of EGaIn measurements is that $J / V$ curves do not require smoothing; these are 'raw' derivatives.) The plots of OPE3, AC and PCP are all U-shaped and slightly asymmetric, reflecting non-resonant tunnelling with somewhat asymmetric
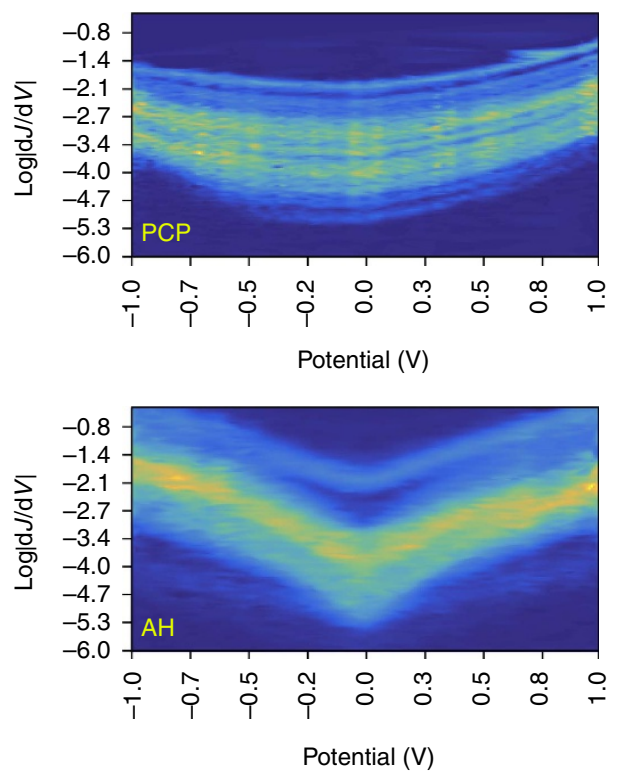

Figure 4 | Differential conductance plots. Conductance heatmap plots of OPE3 and PCP (top left and right, respectively) and AC and AH (bottom left and right, respectively) showing histograms binned to $\log \left|\frac{d}{d V}\right|$ (conductance, $Y$-axis) versus potential (in $V, X$-axis). The colours correspond to the frequencies of the histograms; lighter colours indicate higher frequencies ( $\max \sim 150$ ); the yellow bands are $\sim \frac{\mathrm{d} \mu_{\mathrm{log}}}{\mathrm{d} V}$ (that is, from Fig. 1). All of the plots except for AH are $\mathrm{U}$-shaped, which is a sign of non-resonant tunnelling. The plot for $\mathrm{AH}$ has a sharp dip the centre and negative curvature near $\pm 1 \mathrm{~V}$, which is a sign that a destructive interference feature exists in the bias window. 
coupling. This observation is consistent with recent work comparing $\mathrm{Au} / \mathrm{molecule} / \mathrm{Au}$ and $\mathrm{Ag}^{\mathrm{TS}} / \mathrm{SAM} / / \mathrm{EGaIn}$ junctions ${ }^{38}$. The magnitudes of PCP and AH are also lower than OPE3 and $\mathrm{AC}$, however, the $\mathrm{AH}$ curve is sharp with obvious negative curvature. This difference is direct, experimental evidence of a destructive QI feature very close to $E_{\mathrm{f}}$, supporting the hypothesis that $\mathrm{AH}$ in a SAM adopts a planar conformation closer to that of $\mathrm{AH}$-crystal than to (the minimized, gas-phase conformation of) AH (Fig. 2).

Transport is dominated by states close to $E_{\mathrm{f}}$, but accurately determining the positions of these states in assembled tunnelling junctions is a principal experimental challenge. The only experimental observable for destructive QI is lower conductance, thus claims that QI leads to the observation that a particular structure is less conductive than a reference structure in tunnelling junctions rest entirely on theoretical methods such as those outlined in this paper. However, when the sharp resonance feature of destructive QI is sufficiently close to $E_{\mathrm{f}}$ (in physical junctions) it can appear as a V-shaped differential conductance plot. Next to this work, there are only two other reports of such features appearing, both involving crossconjugated quinone derivatives of $\mathrm{AC}^{37,39}$. In both cases QI features close to $E_{\mathrm{f}}$ appear in DFT calculations for two structures, but appear in differential conductance plots for one. Thus, our work is the first experimental evidence of destructive QI modulated by through-space conjugation and the first observation of conformer-dependent QI. We base our claim that the interference feature $\mathrm{AH}$ of lies near $E_{\mathrm{f}}$ entirely on experimental evidence because it is not possible even to estimate the electrostatics in a metal/SAM/metal junction with the available atomistic and structural information; however, deriving $E_{\mathrm{f}}$ from $V_{\text {trans }}$ gives eminently reasonable relative levelalignments between OPE3/PCP and AC/AH with respect to the HOPS. Given the similarity in length and HOPS between OPE3/ $\mathrm{PCP}$ and $\mathrm{AC} / \mathrm{AH}$ and the differential conductance plots, we can unambiguously ascribe the suppressed conductivity of both PCP and $\mathrm{AH}$ to the destructive $\mathrm{QI}$ in the transmission spectra of PCPcrystal and AH-crystal and conclude that $J$ is dominated by a single conformer and that the through-space elements are better described by X-ray crystal structures than gas-phase optimized structures.

As the complexity of organic structures investigated in tunnelling junctions grows, the details of molecular conformations become more important. And moving from top-down spectroscopic tools towards functional, devicelike platforms ${ }^{2-4,40-44}$ will probably involve bottom-up molecular tunnelling junctions based on $\mathrm{SAMs}^{7}$, in which molecules are in a (liquid) crystalline state. Such junctions represent a form of nanotechnology closest to Nature in that the nanoscopic structure and function are simultaneously and inseparably defined by the equilibrium self-assembly of molecules; differences of $0.06 \AA-0.11 \AA$ can completely suppress QI in DFT simulations. Using QI as a probe, we can separate the effects of interrupted $\pi$ conjugation from those of QI, which are normally conflated experimental observables. And in doing so we provided unambiguous evidence that through-space conjugation can cause interference effects and are not simply 'non-conjugated'. When detailed conformation and packing cannot be determined experimentally, X-ray crystal structures of the pure compounds may be a better approximation of molecular conformation in densely packed SAMs than DFT-minimized structures and transition voltages may provide a reasonable approximation for level-alignment to relate transmission calculations to SAM-based junctions. This approach to understanding transport properties should be generalizable-even in the absence of an observable such as through-space QI-facilitating detailed theoretical and experimental studies on bottom-up, large-area molecular junctions comprising SAMs.

\section{Methods}

Synthesis. The synthesis of AH, AC and OPE3 is described elsewhere ${ }^{18}$. All compounds were stored in nitrogen-flushed vials and in the dark. Their structures were verified by acquiring ${ }^{1} \mathrm{H}-\mathrm{NMR}$ and Fourier transform infrared spectra immediately before use and comparing with the spectra acquired immediately after purification. PCP was prepared starting from $p-2,2$-cyclophane as described in the Supplementary Methods and shown in Supplementary Fig. 18.

Self-assembled monolayers. Care must be taken when forming SAMs from conjugated 'molecular wire' compounds such as these because of the tendency for the deprotected dithiol(ates) to lie flat due to their bidentate structure and favourable $\pi$-Au interactions. SAMs of molecular wires on Au-on-mica were previously formed from a mixture of THF and $\mathrm{Et}_{3} \mathrm{~N}$ (refs 9,19), but in the current work $\mathrm{Au}^{\mathrm{TS}}$ is used, which is supported by optical adhesive and is therefore incompatible with THF. Thus, the procedure was modified and SAMs were formed by incubating the thioacetate precursors with $1 \times 1 \mathrm{~cm}$ template-stripped $\mathrm{Au}$ surfaces ( $100 \mathrm{~nm}$-thick) overnight in $3 \mathrm{ml}$ of $50 \mu \mathrm{M}$ solution of the respective compound in freshly distilled toluene followed by addition of $0.05 \mathrm{ml}$ of $17 \mathrm{mM}$ diazabicycloundec-7-ene solution in toluene $1 \mathrm{~h}$ prior the measurement. The substrates were then rinsed with ethanol and let to dry for $10 \mathrm{~min}$.

To minimize the chance of oxidative damage to the compounds and SAMs, sample preparation, handling and measurement with the EGaIn setup were all performed in a nitrogen flow box with a controlled $\mathrm{O}_{2}$ level between 1-3\% (some $\mathrm{O}_{2}$ is necessary to form tips of EGaIn) and humidity below $10 \%$. At least 20 junctions were measured on each of at least three substrates per molecule (10 scans from $0 \mathrm{~V} \rightarrow 1 \mathrm{~V} \rightarrow-1 \mathrm{~V} \rightarrow 0 \mathrm{~V}$, steps of $0.05 \mathrm{~V}$ ) for a total of at least 600 traces per SAM. A new EGaIn tip was prepared every 5-8 junctions and flattened by gently pushing it on a Si wafer few times according to the procedure reported by Simeone et al. ${ }^{26}$. The details of the EGaIn setup are described elsewhere.

Characterization of the SAMs was performed by HRXPS and NEXAFS spectroscopy. The measurements were carried our at the synchrotron storage ring MAX II at MAX-IV facility in Lund, Sweden and synchrotron radiation facility BESSY II in Berlin, Germany using the bending magnet beamlines D1011 and HE-SGM, respectively. HRXPS data were used to calculate the effective thickness and packing density of the SAMs. NEXAFS data were used to monitor the chemical identity and molecular inclination in the SAMs. See Supplementary Methods for details.

Ellipsometric measurements were acquired in air on a V-Vase Rotating Analyzer equipped with a HS-190 monochromator ellipsometer and calculated via a two-layer model consisting of a bottom Au layer, for which optical constants were calculated from freshly prepared template-stripped Au surfaces, and a Cauchy layer with a chosen value of $n=1.5$ and $k=0$ at all wavelengths $(A=1.5, B=C=0)^{19}$.

Calculations. All DFT calculations were performed using Orca 3.0.3 (ref. 45). Structures were first minimized using BP/Def2-TZV(2d) and then point energies were calculated using B3LYP/Def2-TZV(2d/sp). Single-molecule junctions were constructed by attaching the minimized or X-ray crystal structures to 12 -atom $\mathrm{Au}(111)$ clusters via the terminal sulfur atoms at hexagonal close-pack hollow sites at a distance of $1.75 \AA$ from the centre of the hollow site. Transport calculations were performed with Artaios beta 020914 using B3LYP/G DUNNING-DZP and LANL2/LANLDZ ECPs according to published methods ${ }^{46}$.

Data availability. All of the data used to prepare this manuscript and the Supplementary Information are available upon request.

\section{References}

1. Nerngchamnong, N. et al. The role of van der waals forces in the performance of molecular diodes. Nat. Nanotech. 8, 113-118 (2013).

2. Ferri, V. et al. Light-powered electrical switch based on cargo-lifting azobenzene monolayers. Angew. Chem. Int. Ed. 47, 3407-3409 (2008).

3. Kronemeijer, A. J. et al. Reversible conductance switching in molecular devices Adv. Mater. 20, 1467-1473 (2008).

4. Seo, S., Min, M., Lee, S. M. \& Lee, H. Photo-switchable molecular monolayer anchored between highly transparent and flexible graphene electrodes. Nat. Commun. 4, 1920 (2013).

5. Snider, R. M., Strycharz-Glaven, S. M., Tsoi, S. D., Erickson, J. S. \& Tender, L. M. Long-range electron transport in geobacter sulfurreducens biofilms is redox gradient-driven. Proc. Natl Acad. Sci. USA 109, 15467-15472 (2012).

6. Love, J., Estroff, L., Kriebel, J. K., Nuzzo, R. \& Whitesides, G. M. Self-assembled monolayers of thiolates on metals as a form of nanotechnology. Chem. Rev. 105, 1103-1170 (2005).

7. Zhang, Y., Zhao, Z., Fracasso, D. \& Chiechi, R. C. Bottom-up molecular tunneling junctions formed by self-assembly. Isr. J. Chem. 54, 513-533 (2014). 
8. Solomon, G. Handbook of Single-Molecule Electronics (Pan Stanford Publishing, 2015).

9. Fracasso, D., Valkenier, H., Hummelen, J. C., Solomon, G. C. \& Chiechi, R. Evidence for quantum interference in sams of arylethynylene thiolates in tunneling junctions with eutectic ga-in (egain) top-contacts. J. Am. Chem. Soc. 133, 9556-9563 (2011).

10. Perrin, M. L. et al. Large negative differential conductance in single-molecule break junctions. Nat. Nanotech. 9, 830-834 (2014).

11. Solomon, G. C., Herrmann, C., Vura-Weis, J., Wasielewski, M. R. \& Ratner, M. A. The chameleonic nature of electron transport through $\pi$-stacked systems. J. Am. Chem. Soc. 132, 7887-7889 (2010).

12. Xiang, D., Jeong, H., Lee, T. \& Mayer, D. Mechanically controllable break junctions for molecular electronics. Adv. Mater. 25, 4845-4867 (2013).

13. Frisenda, R., Janssen, V. A. E. C., Grozema, F. C., van der Zant, H. S. J. \& Renaud, N. Mechanically controlled quantum interference in individualstacked dimers. Nat. Chem. 8, 1099-1104 (2016).

14. Weiss, E. A. et al. Influence of defects on the electrical characteristics of mercury-drop junctions: self-assembled monolayers of N-alkanethiolates on rough and smooth silver. J. Am. Chem. Soc. 129, 4336-4349 (2007).

15. Lykkebo, J., Gagliardi, A., Pecchia, A. \& Solomon, G. C. Iets and quantum interference: propensity rules in the presence of an interference feature. J. Phys. Chem. 141, 124119 (2014)

16. Rissner, F. et al. Collectively induced quantum-confined stark effect in monolayers of molecules consisting of polar repeating units. J. Am. Chem. Soc. 133, 18634-18645 (2011).

17. Chiechi, R. C., Weiss, E. A., Dickey, M. D. \& Whitesides, G. M. Eutectic gallium-indium (egain): a moldable liquid metal for electrical characterization of self-assembled monolayers. Angew. Chem. Int. Ed. 120, 148-150 (2008).

18. Valkenier, H. et al. Cross-conjugation and quantum interference: a general correlation? Phys. Chem. Chem. Phys. 16, 653-662 (2014)

19. Valkenier, H. et al. Formation of high-quality self-assembled monolayers of conjugated dithiols on gold: base matters. J. Am. Chem. Soc. 133, 4930-4939 (2011).

20. Tai, Y. et al. Fabrication of thiol-terminated surfaces using aromatic selfassembled monolayers. J. Phys. Chem. B 108, 16806-16810 (2004).

21. Yuan, L., Jiang, L., Thompson, D. \& Nijhuis, C. A. On the remarkable role of surface topography of the bottom electrodes in blocking leakage currents in molecular diodes. J. Am. Chem. Soc. 136, 6554-6557 (2014).

22. Chen, J., Wang, Z., Oyola-Reynoso, S., Gathiaka, S. M. \& Thuo, M. Limits to the effect of substrate roughness or smoothness on the odd-even effect in wetting properties of n-alkanethiolate monolayers. Langmuir 31, 7047-7054 (2015).

23. Kong, G. D., Kim, M., Cho, S. J. \& Yoon, H. J. Gradients of rectification: Tuning molecular electronic devices by the controlled use of different-sized diluents in heterogeneous self-assembled monolayers. Angew. Chem. Int. Ed. 55, 10307-10311 (2016)

24. Schneebeli, S. T. et al. Single-molecule conductance through multiple $\pi$ - $\pi$-stacked benzene rings determined with direct electrode-to-benzene ring connections. J. Am. Chem. Soc. 133, 2136-2139 (2011).

25. Van Dyck, C. \& Ratner, M. A. Molecular rectifiers: a new design based on asymmetric anchoring moieties. Nano Lett. 15, 1577-1584 (2015).

26. Simeone, F. C. et al. Defining the value of injection current and effective electrical contact area for egain-based molecular tunneling junctions. J. Am. Chem. Soc. 135, 18131-18144 (2013).

27. Heimel, G., Romaner, L., Zojer, E. \& Bredas, J.-L. The interface energetics of self-assembled monolayers on metals. Acc. Chem. Res. 41, 721-729 (2008).

28. Verwster, E., Hofmann, O. T., Egger, D. A. \& Zojer, E. Electronic properties of biphenylthiolates on au(111): the impact of coverage revisited. J. Phys. Chem. C 119, 7817-7825 (2015).

29. Heimel, G., Romaner, L., Brdas, J.-L. \& Zojer, E. Interface energetics and level alignment at covalent metal-molecule junctions: $\pi$-conjugated thiols on gold. Phys. Rev. Lett. 96, 196806 (2006).

30. Egger, D. A. \& Zojer, E. Anticorrelation between the evolution of molecular dipole moments and induced work function modifications. J. Phys. Chem. Lett. 4, 3521-3526 (2013)

31. Kovalchuk, A. et al. Transition voltages respond to synthetic reorientation of embedded dipoles in self-assembled monolayers. Chem. Sci. 7, 781-787 (2016).

32. Alloway, D. M. et al. Interface dipoles arising from self-assembled monolayers on gold: uv-photoemission studies of alkanethiols and partially fluorinated alkanethiols. J. Phys. Chem. B 107, 11690-11699 (2003).

33. Beebe, J. M., Kim, B., Frisbie, C. D. \& Kushmerick, J. G. Measuring relative barrier heights in molecular electronic junctions with transition voltage spectroscopy. ACS Nano 2, 827-832 (2008).

34. Seferos, D. S., Blum, A. S., Kushmerick, J. G. \& Bazan, G. C. Single-molecule charge-transport measurements that reveal technique-dependent perturbations. J. Am. Chem. Soc. 128, 11260-11267 (2006)
35. Seferos, D. S., Trammell, S. A., Bazan, G. C. \& Kushmerick, J. G. Probing $\pi$-coupling in molecular junctions. Proc. Natl Acad. Sci. USA 102, 8821-8825 (2005).

36. Chen, L. et al. Multichannel conductance of folded single-molecule wires aided by through-space conjugation. Angew. Chem. Int. Ed. 54, 4231-4235 (2015).

37. Gudon, C. M. et al. Observation of quantum interference in molecular charge transport. Nature Nanotech. 7, 305-309 (2012).

38. Garrigues, A. R. et al. A single-level tunnel model to account for electrical transport through single molecule- and self-assembled monolayer-based junctions. Sci. Rep. 6, 26517 (2016).

39. Rabache, V. et al. Direct observation of large quantum interference effect in anthraquinone solid-state junctions. J. Am. Chem. Soc. 135, 10218-10221 (2013).

40. van Hal, P. A. et al. Upscaling, integration and electrical characterization of molecular junctions. Nat. Nanotech. 3, 749-754 (2008).

41. Nijhuis, C. A., Reus, W. F., Siegel, A. C. \& Whitesides, G. M. A molecular half-wave rectifier. J. Am. Chem. Soc. 133, 15397-15411 (2011).

42. Pourhossein, P. \& Chiechi, R. C. Directly addressable sub-3 nm gold nanogaps fabricated by nanoskiving using self-assembled monolayers as templates. ACS Nano 6, 5566-5573 (2012).

43. Li, T. et al. Ultrathin reduced graphene oxide films as transparent top-contacts for light switchable solid-state molecular junctions. Adv. Mater. 25, 4164-4170 (2013).

44. Wan, A., Jiang, L., Sangeeth, C. S. S. \& Nijhuis, C. A. Reversible soft topcontacts to yield molecular junctions with precise and reproducible electrical characteristics. Adv. Funct. Mater. 24, 4442-4456 (2014).

45. Neese, F. The orca program system. Wiley Interdiscip. Rev. Comput. Mol. Sci. 2, 73-78 (2012)

46. Herrmann, C., Solomon, G. C., Subotnik, J. E., Mujica, V. \& Ratner, M. A. Ghost transmission: how large basis sets can make electron transport calculations worse. J. Chem. Phys. 132, 024103 (2010).

\section{Acknowledgements}

R.C.C., M.C. and A.K. acknowledge the European Research Council for the ERC Starting Grant 335473 (MOLECSYNCON). T.W. and M.Z. thank the Max-IV facility and Helmholtz-Zentrum Berlin for the allocation of beamtime and the technical support during the experiments. Part of this work was supported financially by the Deutsche Forschungsgemeinschaft (grants ZH 63/14-2). We thank the Center for Information Technology of the University of Groningen for their support of the Peregrine high performance computing cluster.

\section{Author contributions}

M.C. and A.K. synthesized and characterized the compounds, carried out the EGaIn and ellipsometry measurements and acquired the X-ray crystal structures. M.C. performed the Greens function and tight-binding calculations. T.W. and M.Z. carried out all synchrotron measurements and related data handling and interpretation. X.Q. performed the CP-AFM measurements. R.C.C. performed the DFT calculations and supervised the project. All the authors contributed to preparing figures and writing the manuscript.

\section{Additional information}

Supplementary Information accompanies this paper at http://www.nature.com/ naturecommunications

Competing financial interests: The authors declare no competing financial interests

Reprints and permission information is available online at http://npg.nature.com/ reprintsandpermissions/

How to cite this article: Carlotti, M. et al. Conformation-driven quantum interference effects mediated by through-space conjugation in self-assembled monolayers. Nat. Commun. 7, 13904 doi: 10.1038/ncomms13904 (2016).

Publisher's note: Springer Nature remains neutral with regard to jurisdictional claims in published maps and institutional affiliations.

This work is licensed under a Creative Commons Attribution 4.0 International License. The images or other third party material in this article are included in the article's Creative Commons license, unless indicated otherwise in the credit line; if the material is not included under the Creative Commons license, users will need to obtain permission from the license holder to reproduce the material. To view a copy of this license, visit http://creativecommons.org/licenses/by/4.0/

(C) The Author(s) 2016 